\title{
Conflict-free Replicated Data Types
}

\author{
Nuno Preguiça \\ DI, FCT, Universidade NOVA de Lisboa and NOVA LINCS, Portugal \\ Carlos Baquero \\ HASLab / INESC TEC \& Universidade do Minho, Portugal \\ Marc Shapiro \\ Sorbonne-Université \& Inria, Paris, France
}

20 February 2018

\section{Definition}

A conflict-free replicated data type (CRDT) is an abstract data type, with a well defined interface, designed to be replicated at multiple processes and exhibiting the following properties: (i) any replica can be modified without coordinating with another replicas; (ii) when any two replicas have received the same set of updates, they reach the same state, deterministically, by adopting mathematically sound rules to guarantee state convergence.

\section{Overview}

Internet-scale distributed systems often replicate data at multiple geographic locations to provide low latency and high availability, despite outages and network failures. To this end, these systems must accept updates at any replica, and propagate these updates asynchronously to the other replicas. This approach allows

replicas to temporarily diverge and requires a mechanism for merging concurrent 
updates into a common state. CRDTs provide a principled approach to address this problem.

As any abstract data type, a CRDT implements some given functionality and exposes a well defined interface. Applications interact with the CRDT only through this interface. As CRDTs are specially designed to be replicated and to allow uncoordinated updates, a key aspect of a CRDT is its semantics in the presence of concurrency. The concurrency semantics defines what is the behavior of the object in the presence of concurrent updates, defining the state of the object for any given set of received updates.

An application developer uses the CRDT interface and concurrency semantics to reason about the behavior of her applications in the presence of concurrent updates. A system developer that needs to create a system that provides CRDTs needs to focus on another aspect of CRDTs: the synchronization model. The synchronization model defines the requirements that the system must meet so that CRDTs work correctly. We now detail each of these aspects independently.

\section{Concurrency semantics}

The operations defined in a data-type may intrinsically commute or not. Consider for instance a Counter data type, a shared integer that supports increment and decrement operations. As these operations commute (i.e., executing them in any order yields the same result) the Counter data type naturally converges towards the expected result. In this case, it is natural that the state of a CRDT object reflects all executed operations.

Unfortunately, for most data-types, this is not the case and several concurrency semantics are reasonable, with different semantics being suitable for different applications. For instance, consider a shared memory cell supporting the assignment operation. If the initial value is 0 , the correct outcome for concurrently assigning 1 and 2 is not well defined.

When defining the concurrency semantics, an important concept that is often used is that of the happens-before relation (Lamport 1978). In a distributed system, an event $e_{1}$ happened-before an event $e_{2}, e_{1} \prec e_{2}$, iff: (i) $e_{1}$ occurred before $e_{2}$ in 
the same process; or (ii) $e_{1}$ is the event of sending message $m$, and $e_{2}$ is the event of receiving that message; or (iii) there exists an event $e$ such that $e_{1} \prec e$ and $e \prec e_{2}$. When applied to CRDTs, we can say that an update $u_{1}$ happened-before an update $u_{2}$ iff the effects of $u_{1}$ had been applied in the replica where $u_{2}$ was executed initially.

As an example, if an event was "Alice reserved the meeting room" it is relevant to know if that was known when "Bob reserved the meeting room" to determine if Alice should be given priority or if two users concurrently tried to reserve the same room.

For instance, let us use happened-before to define the semantics of the add-wins set (also known as observed-remove set, OR-set (Shapiro et al 2011)). Intuitively, in the add-wins semantics, in the presence of two operations that do not commute, a concurrent add and remove of the same element, the add wins leading to a state where the element belongs to the set. More formally, the set interface has two update operations: (i) $\operatorname{add}(e)$, for adding element $e$ to the set; and (ii) $\operatorname{rmv}(e)$, for removing element $e$ from the set. Given a set of update operations $O$ that are related by the happens before partial order $\prec$, the state of the set is defined as: $\{e \mid \operatorname{add}(e) \in O \wedge \nexists \operatorname{rmv}(e) \in O \cdot \operatorname{add}(e) \prec \operatorname{rmv}(e)\}$.

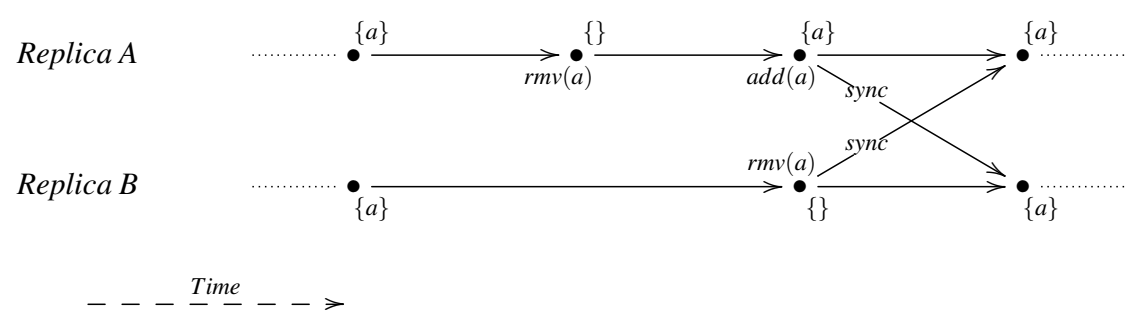

Figure 1: Run with an add-wins set.

Figure 1 shows a run where an add-wins set is replicated in two replicas, with initial state $\{a\}$. In this example, in replica A, $a$ is first removed and later added again to the set. In replica B, $a$ is removed from the set. After receiving the updates from the other replica, both replicas end up with element $a$ in the set. The reason for this is that there is no $r m v(a)$ that happened after the $a d d(a)$ executed in replica 
A.

An alternative semantics based on the happens-before relation is the removewins. Intuitively, in the remove-wins semantics, in the presence of a concurrent add and remove of the same element, the remove wins leading to a state where the element is not in the set. More formally, given a set of update operations $O$, the state of the set is defined as: $\{e \mid \operatorname{add}(e) \in O \wedge \forall \operatorname{rmv}(e) \in O \cdot \operatorname{rmv}(e) \prec \operatorname{add}(e)\}$. In the previous example, after receiving the updates from the other replica, the state of both replicas would be the empty set, because there is no $\operatorname{add}(a)$ that happened after the $r m v(a)$ in replica B.

Another relation that can be useful for defining the concurrency semantics is that of a total order among updates and particularly a total order that approximates wall-clock time. In distributed systems, it is common to maintain nodes with their physical clocks loosely synchronized. When combining the clock time with a site identifier, we have unique timestamps that are totally ordered. Due to the clock skew among multiple nodes, although these timestamps approximate an ideal global physical time, they do not necessarily respect the happens-before relation. This can be achieved by combining physical and logical clocks, as shown by Hybrid Logical Clocks (Kulkarni et al 2014), or by only arbitrating a wall-clock total order for the events that are concurrent under causality (Zawirski et al 2016).

This relation allows to define the last-writer-wins semantics, where the value written by the last writer wins over the values written previously, according to the defined total order. More formally, with the set $O$ of operations now totally ordered by $<$, the state of a last-writer-wins set would be defined as: $\{e \mid \operatorname{add}(e) \in O \wedge$ $\forall \operatorname{rmv}(e) \in O \cdot \operatorname{rmv}(e)<\operatorname{add}(e)\}$. Returning to our previous example, the state of the replicas after the synchronization would include $a$ if, according the total order defined among the operations, the $r m v(a)$ of replica B is smaller than the $\operatorname{add}(a)$ of replica A. Otherwise, the state would be the empty set.

We now briefly introduce the concurrency semantics proposed for several CRDTs. 


\section{Set}

For a set CRDT, we have shown the difference between three possible concurrency semantics: add-wins, remove-wins and last-writer-wins.

\section{Register}

A register CRDT maintains an opaque value and provides a single update operation that writes an arbitrary value: wr(value). Two concurrency semantics have been proposed leading to two different CRDTs: the multi-value register and the lastwriter-wins register. In the multi-value register, all concurrently written values are kept. In this case, the read operation return the set of concurrently written values. Formally, the state of a multi-value register is defined as the multi-set: $\{v \mid \mathrm{wr}(v) \in O \wedge \nexists \operatorname{wr}(u) \in O \cdot \operatorname{wr}(v) \prec \operatorname{wr}(u)\}$.

In the last-writer-wins register, only the value of the last write is kept, if any. Formally, the state of a last-writer-wins register can be defined as a set that is either empty or holds a single value: $\{v \mid \operatorname{wr}(v) \in O \wedge \nexists \operatorname{wr}(u) \in O \cdot \operatorname{wr}(v)<\operatorname{wr}(u)\}$.

\section{Counter}

A counter CRDT maintains an integer and can be modified by update operations inc and dec, to increase and decrease by one unit its value, respectively (this can easily generalize to arbitrary amounts). As mentioned previously, as operations intrinsically commute, the natural concurrency semantics is to have a final state that reflects the effects of all registered operations. Thus the result state is obtained by counting the number of increments and subtracting the number of decrements: $\mid\{$ inc $\mid$ inc $\in O\}|-|\{\operatorname{dec} \mid \operatorname{dec} \in O\} \mid$.

Now consider that we want to add a write operation $\operatorname{wr}(n)$, to update the value of the counter to a given value. This opens two questions related with the concurrency semantics. First, what should be the final state when two concurrent write operations are executed. In this case, the last-writer-wins semantics would be simple (as maintaining multiple values, as in the multi-value register, is overly complex). 
Second, what is the result when concurrent writes and inc/dec operations are executed. In this case, by building on the happens-before relation, we can define several concurrency semantics. One possibility is a write-wins semantics, where inc/dec operations have no effect when executed concurrently with the last write. Formally, for a given set $O$ of updates that include at least a write operation, let $v$ be the value in the last write, i.e., $\operatorname{wr}(v) \in O \wedge \nexists \operatorname{wr}(u) \in O \cdot \operatorname{wr}(v)<\operatorname{wr}(u)$. The value of the counter would be $v+o$, with $o=\mid$ inc $\mid$ inc $\in O \wedge \operatorname{wr}(v) \prec$ inc $\} \mid-$ $|\{\operatorname{dec} \mid \operatorname{dec} \in O \wedge \operatorname{wr}(v) \prec \operatorname{dec}\}|$ representing inc/dec operations that happened after the last write.

\section{Other CRDTs}

A number of other CRDTs have been proposed in literature, including CRDTs for elementary data structures, such as Lists (Preguiça et al 2009; Weiss et al 2009; Roh et al 2011), Maps (Brown et al 2014; Almeida et al 2018) and Graphs (Shapiro et al 2011), and more complex structures, such as JSON documents (Kleppmann and Beresford 2017). For each of these CRDTs, the developers have defined and implemented a type-specific concurrency semantics.

\section{Synchronization Model}

A replicated system needs to synchronize its replicas, by propagating and applying updates in every replica. There are two main approaches to propagate updates: state-based and operation-based replication.

In state-based replication, replicas synchronize by establishing bi-directional (or unidirectional) synchronization sessions, where both (one, resp.) replicas send their state to a peer replica. When a replica receives the state of a peer, it merges the received state with its local state. As long as the synchronization graph is connected, every update will eventually propagate to all replicas.

CRDTs designed for state-based replication define a merge function to integrate the state of a remote replica. It has been shown (Shapiro et al 2011) that all replicas of a CRDT converge if: (i) the states of the CRDT are partially ordered according to $\leq$ forming a join semilattice; (ii) an operation modifies the state $s$ of a 
replica by an inflation, producing a new state that is larger or equal to the original state according to $\leq$, i.e., for any operation $m, s \leq m(s)$; (iii) the merge function produces the join (least upper bound) of two states, i.e. for states $s, u$ it derives $s \sqcup u$.

In operation-based replication, replicas converge by propagating operations to every other replica. When an operation is received in a replica, it is applied to the local replica state. Besides requiring that every operation is reliably delivered to all replicas, e.g. by using some reliable multicast communication subsystem, some systems may require operations to be delivered according to some specific order, with causal order being the most common.

CRDTs designed for operation-based replication must define, for each operation, a generator and an effector function. The generator function executes in the replica where the operation is submitted, it has no side-effects and generates a effector that encodes the side-effects of the operation. In other words, the effector is a closure created by the generator depending on the state of the origin replica. The effector operation must be reliably executed in all replicas, where it updates the replica state. Shapiro et al (2011) show that if effector operations are delivered in causal order, replicas will converge to the same state if concurrent effector operations commute. If effector operations may be delivered without respecting causal order, then all effector operations must commute. Most operation-based CRDT design require causal delivery.

Alternative models: When operations modify only part of the state, propagating the complete state for synchronization to a remote replica is inefficient, as the remote replica already knows most of the state. Delta-state CRDTs (Almeida et al 2018) address this issue by propagating only delta-mutators, that encode the changes that have been made to a replica since the last communication. The first time a replica communicates with some other replica, the full state needs to be propagated. This can be further improved, as shown in big delta state CRDTs (van der Linde et al 2016), typically at the cost of storing more metadata in the CRDT state. Another improvement is to compute digests that help determine which parts of a remote state are needed, avoiding shipping full states (Enes 2017).

In the context of operation-based replication, effector operations should be ap- 
plied immediately in the source replica, that executed the generator. However, propagation to other replicas can be deferred for some period and effectors stored in an outbound log, presenting an opportunity to compress the log by rewriting some operations - e.g. two add(1) operations in a counter can be converted in a add(2) operation. This mechanism has been used by Cabrita et. al. (Cabrita and Preguiça 2017). Delta-mutators can also be seen as a compressed representation of a $\log$ of operations.

The operation-based CRDTs require executing a generator function against the replica state to compute an effector operation. In some scenarios, this may introduce an unacceptable delay for propagating an operation. Pure-operation based CRDTs (Baquero et al 2014) address this issue by allowing the original operations to be propagated to all replicas, typically at the cost of more complex operations and of having to store more metadata in the CRDT state.

\section{Key research findings}

\section{Preservation of sequential semantics}

When modeling an abstract data type that has an established semantics under sequential execution, CRDTs should preserve that semantics. For instance, CRDT sets should ensure that if the last operation in a sequence of operations to a set added a given element, then a query operation immediately after that one will show the element to be present on the set. Conversely, if the last operation removed an element, then a subsequent query should not show its presence.

Sequential execution can occur even in distributed settings if synchronization is frequent. An instance can be updated in replica A, merged into another replica $\mathrm{B}$ and updated there, and merged back into replica A before A tries to update it again. In this case we have a sequential execution, even though updates have been executed in different replicas.

Historically, not all CRDT designs have met this property. The two-phase set CRDT (2PSet), does not allow re-adding an element that was removed, and thus it breaks the common sequential semantics. Later CRDT set designs, such as add- 
wins and remove-wins sets, do preserve the original sequential semantics while providing different arbitration orders to concurrent operations.

\section{Extended behaviour under concurrency}

Some CRDT designs handle concurrent operations by arbitrating a given sequential ordering to accommodate concurrent execution. For example, the state of a last-writer-wins set replica shown by its interface can be explained by a sequential execution of the operations according to the LWW total order used. When operations commute, such as in G-Counters and PN-Counters, there might even be several sequential executions that explain a given state.

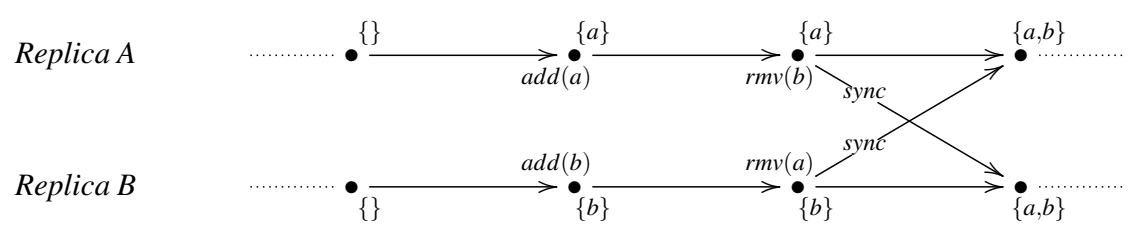

Figure 2: Add-wins set run showing that there might be no sequential execution of operations that explains CRDTs behavior.

Not all CRDTs need or can be explained by sequential executions. The addwins set is an example of a CRDT where there might be no sequential execution of operations to explain the state observed, as Figure 2 shows. In this example, the state of the set after all updates propagate to all replicas includes $a$ and $b$, but in any sequential extension of the causal order a remove operation would always be the last operation, and consequently the removed element could not belong to the set.

Some other CRDTs can exhibit states that are only attained when concurrency does occur. An example is the multi-value register, a register that supports a simple write and read interface. If used sequentially, sequential semantics is preserved, and a read will show the outcome of the most recent write in the sequence. However if two or more value are written concurrently, the subsequent read will show all those values (as the multi-value name implies), and there is no sequential execu- 
tion that can explain this result. We also note that a follow up write can overwrite both a single value and multiple values.

\section{Guaranties and limitations}

An important property of CRDTs is that an operation can always be accepted at any given replica and updates are propagated asynchronously to other replicas. In the CAP theorem framework (Brewer 2010; Gilbert and Lynch 2002), the CRDT conflict-free approach favors availability over consistency when facing communication disruptions. This leads to resilience to network failure and disconnection, since no prior coordination with other replicas is necessary before accepting an operation. Further, operations can be accepted with minimal user perceived latency since they only require local durability. By eschewing global coordination, replicas evolve independently and reads will not reflect operations accepted in remote replicas that have not yet been propagated to the local replica.

In the absence of global coordination, session guaranties (Terry et al 1994) specify what the user applications can expect from their interaction with the system's interface. Both state based CRDTs, and operation based CRDTs when supported by reliable causal delivery, provide per-object causal consistency. Thus, in the context of a given replicated object, the traditional session guaranties are met. CRDT based systems that lack transactional support can enforce system-wide causal consistency, by integrating multiple objects in a single map/directory object (Almeida et al 2018). Another alternative is to use mergeable transactions to read from a causally-consistent database snapshot and to provide write atomicity (Preguiça et al 2014).

Some operations cannot be expressed in a conflict free framework and will require global agreement. As an example, in an auction system, bids can be collected under causal consistency, and a new bid will only have to increase the offer with respect to bids that are known to causally precede it. However, closing the auction and selecting a single winning bid will require global agreement. It is possible to design a system that integrates operations with different coordination requirements and only resorts to global agreement when necessary (Li et al 2012; Sovran et al 
2011).

Some global invariants, that usually are enforced with global coordination, can be enforced in a conflict free manner by using escrow techniques (O'Neil 1986) that split the available resources by the different replicas. For instance, the Bounded Counter CRDT (Balegas et al 2015b) defines a counter that never goes negative, by assigning to each replica a number of allowed decrements under the condition that the sum of all allowed decrements do not exceed the value of the counter. While its assigned decrements are not exhausted, replicas can accept decrements without coordinating with other replicas. After a replica exhaust its allowed decrements, a new decrement will either fail or require synchronizing with some replica that still can decrement. This technique uses point to point coordination, and can be generalized to enforce other system wide invariants (Balegas et al 2015a).

\section{Examples of applications}

CRDTs have been used in a large number of distributed systems and applications that adopt weak consistency models. The adoption of CRDTs simplifies the development of these systems and applications, as CRDTs guarantee that replicas converge to the same state when all updates are propagated to all replicas. We can group the systems and applications that use CRDTs into two groups: storage systems that provide CRDTs as their data model; and applications that embed CRDTs to maintain their internal data.

CRDTs have been integrated in several storage systems that make them available to applications. An application uses these CRDTs to store their data, being the responsibility of the storage systems to synchronize the multiple replicas. The following commercial systems use CRDT: Riak, ${ }^{1}$ Redis (Biyikoglu 2017) and Akka. ${ }^{2}$ A number of research prototypes have also used CRDT, including Walter (Sovran

\footnotetext{
${ }^{1}$ Developing with Riak KV Data Types http://docs.basho.com/riak/kv/2.2.3/developing/ data-types/.

${ }^{2}$ Akka Distributed Data: https://doc.akka.io/docs/akka/2.5.4/scala/distributed-data.html.
} 
et al 2011), SwiftCloud (Preguiça et al 2014) and Antidote ${ }^{3}$ (Akkoorath et al 2016).

CRDTs have also been embedded in multiple applications. In this case, developers either used one of the available CRDT libraries, implemented themselves some previously proposed design or designed new CRDTs to meet their specific requirements. An example of this latter use is Roshi ${ }^{4}$, a LWW-element-set CRDT used for maintaining an index in SoundCloud stream.

\section{Future directions of research}

\section{Scalability}

In order to track concurrency and causal predecessors, CRDT implementations often store metadata that grows linearly with the number of replicas (Charron-Bost 1991). While global agreement suffers from greater scalability limitations since replicas must coordinate to accept each operation, the metadata cost from causality tracking can limit the scalability of CRDTs when aiming for more than a few hundred replicas. A large metadata footprint can also impact on the computation time of local operations, and will certainly impact the required storage and communication.

Possible solutions can be sought in more compact causality representations when multiple replicas are synchronized among the same nodes (Malkhi and Terry 2007; Preguiça et al 2014; Gonçalves et al 2017) or by hierarchical approaches that restrict all to all synchronization and enable more compact mechanisms (Almeida and Baquero 2013).

\section{Reversible computation}

Non trivial Internet services require the composition of multiple sub-systems, to provide storage, data dissemination, event notification, monitoring and other needed components. When composing sub-systems, that can fail independently or simply

\footnotetext{
${ }^{3}$ Antidote:http://antidotegb.org/.

${ }^{4}$ Roshi is a large-scale CRDT set implementation for timestamped events https://github.com/ soundcloud/roshi.
} 
reject some operations, it is useful to provide a CRDT interface that undoes previously accepted operations. Another scenario that would benefit from undo is collaborative editing of shared documents, where undo is typically a feature available to users.

Undoing an increment on a counter CRDT can be achieved by a decrement. Logoot-Undo (Weiss et al 2010) proposes a solution for undoing (and redoing) operations for a sequence CRDT used for collaborative editing. However providing an uniform approach to undoing, reversing, operations over the whole CRDT catalog is still an open research direction. The support of undo is also likely to limit the level of compression that can be applied to CRDT metadata.

\section{Security}

While access to a CRDT based interface can be restricted by adding authentication, any accessing replica has the potential to issue operations that can interfere with the other replicas. For instance, delete operations can remove all existing state. In state based CRDTs, replicas have access to state that holds a compressed representation of past operations and metadata. By manipulation of this state and synchronizing to other replicas, it is possible to introduce significant attacks to the system operation and even its future evolution.

Applications that store state on third party entities, such as in cloud storage providers, might elect not to trust the provider and choose end-to-ends encryption of the exchanged state. This, however, would require all processing to be done at the edge, under the application control. A research direction would be to allow some limited form of computation, such as merging state, over information whose content is subject to encryption. Potential techniques, such as homomorphic encryption, are likely to pose significant computational costs. An alternative is to execute operations in encrypted data without disclosing it, relying on specific hardware support, such as Intel SGX and ARM TrustZone. 


\section{Non-uniform replicas}

The replication of CRDTs typically assumes that eventually all replicas will reach the same state, storing exactly the same data. However, depending on the read operations available in the CRDT interface, it might not be necessary to maintain the same state in all replicas. For example, an object that has a single read operation returning the top-K elements added to the object only needs to maintain those top$\mathrm{K}$ elements in every replica. The remaining elements are necessary if a remove operation is available, as one of the elements not in the top needs to be promoted when a top element is removed. Thus, each replica can maintain only the top-K elements and the elements added locally.

This replication model is named non-uniform replication (Cabrita and Preguiça 2017) and can be used to design CRDTs that exhibit important storage and bandwidth savings when compared with alternatives that keep all data in all replicas. Although it is clear that this model cannot be used for all data types, several useful CRDT design have been proposed, including top-K, top-Sum and histogram. To understand what data types can adopt this model and how to explore it in practice is an open research question.

\section{Verification}

An important aspect related with the development of distributed systems that use CRDTs is the verification of the correctness of the system. This involves not only verifying the correctness of CRDT designs, but also the correctness of the system that uses CRDTs. A number of works have addressed these issues.

Regarding the verification of the correctness of CRDTs, several approaches have been taken. The most commonly used approach is to have proofs when designs are proposed or to use some verification tools for the specific data type, such as TLA (Lamport 1994) or Isabelle ${ }^{5}$. There has also been some works that proposed general techniques for the verification of CRDTs (Burckhardt et al 2014; Zeller et al 2014; Gomes et al 2017), which can be used by CRDT developers to verify the correctness of their designs. Some of these works (Zeller et al 2014;

\footnotetext{
${ }^{5}$ Isabelle: http://isabelle.in.tum.de/.
} 
Gomes et al 2017) include specific frameworks that help the developer in the verification process.

A number of other works have proposed techniques to verify the correctness of distributed systems that use CRDTs (Gotsman et al 2016; Zeller 2017; Balegas et al 2015a). These works typically require the developer to specify the properties that the distributed system must maintain, and a specification of the operations in the system (that is independent of the actual code of the system). Despite these works, the verification of the correctness of CRDT designs and of systems that use CRDTs, how these verification techniques can be made available to programmers, and how to verify the correctness of implementations, remain an open research problem.

\section{Acknowledgments}

This work was partially supported by NOVA LINCS (UID/CEC/04516/2013), EU H2020 LightKone project (732505), and SMILES line in project TEC4Growth (NORTE-01-0145-FEDER-000020).

\section{References}

Akkoorath DD, Tomsic AZ, Bravo M, Li Z, Crain T, Bieniusa A, Preguiça N, Shapiro M (2016) Cure: Strong semantics meets high availability and low latency. In: Proceedings of the 2016 IEEE 36th International Conference on Distributed Computing Systems (ICDCS), pp 405-414, DOI 10.1109/ICDCS.2016. 98

Almeida PS, Baquero C (2013) Scalable eventually consistent counters over unreliable networks. CoRR abs/1307.3207, URL http://arxiv.org/abs/1307.3207, 1307.3207

Almeida PS, Shoker A, Baquero C (2018) Delta state replicated data types. J Parallel Distrib Comput 111:162-173, DOI 10.1016/j.jpdc.2017.08.003, URL https://doi.org/10.1016/j.jpdc.2017.08.003 
Balegas V, Duarte S, Ferreira C, Rodrigues R, Preguiça NM, Najafzadeh M, Shapiro M (2015a) Putting consistency back into eventual consistency. In: Réveillère L, Harris T, Herlihy M (eds) Proceedings of the Tenth European Conference on Computer Systems, EuroSys 2015, Bordeaux, France, April 21-24, 2015, ACM, pp 6:1-6:16, DOI 10.1145/2741948.2741972, URL http: //doi.acm.org/10.1145/2741948.2741972

Balegas V, Serra D, Duarte S, Ferreira C, Shapiro M, Rodrigues R, Preguiça NM (2015b) Extending eventually consistent cloud databases for enforcing numeric invariants. In: 34th IEEE Symposium on Reliable Distributed Systems, SRDS 2015, Montreal, QC, Canada, September 28 - October 1, 2015, IEEE Computer Society, pp 31-36, DOI 10.1109/SRDS.2015.32, URL https: //doi.org/10.1109/SRDS.2015.32

Baquero C, Almeida PS, Shoker A (2014) Making Operation-based CRDTs Operation-based. In: Proceedings of the First Workshop on Principles and Practice of Eventual Consistency, ACM, New York, NY, USA, PaPEC '14, pp 7:1-7:2, DOI 10.1145/2596631.2596632, URL http://doi.acm.org/10.1145/ 2596631.2596632

Biyikoglu C (2017) Under the hood: Redis crdts (conflict-free replicated data types). Online (accessed 24-Nov-2017) https://goo.gl/tGqU7h

Brewer E (2010) On a certain freedom: exploring the CAP space, invited talk at PODC 2010, Zurich, Switzerland

Brown R, Cribbs S, Meiklejohn C, Elliott S (2014) Riak DT Map: A Composable, Convergent Replicated Dictionary. In: Proceedings of the First Workshop on Principles and Practice of Eventual Consistency, ACM, New York, NY, USA, PaPEC '14, pp 1:1-1:1, DOI 10.1145/2596631.2596633, URL http://doi.acm. org/10.1145/2596631.2596633

Burckhardt S, Gotsman A, Yang H, Zawirski M (2014) Replicated data types: Specification, verification, optimality. In: Proceedings of the 41st ACM SIGPLAN-SIGACT Symposium on Principles of Programming Languages, 
ACM, New York, NY, USA, POPL '14, pp 271-284, DOI 10.1145/2535838. 2535848, URL http://doi.acm.org/10.1145/2535838.2535848

Cabrita G, Preguiça N (2017) Non-uniform Replication. In: Proceedings of the 21th International Conference on Principles of Distributed Systems, OPODIS 2017, Schloss Dagstuhl - Leibniz-Zentrum fuer Informatik, LIPIcs

Charron-Bost B (1991) Concerning the size of logical clocks in distributed systems. Inf Process Lett 39(1):11-16, DOI 10.1016/0020-0190(91)90055-M, URL https://doi.org/10.1016/0020-0190(91)90055-M

Enes V (2017) Efficient Synchronization of State-based CRDTs. Master's thesis, Universidade do Minho, URL vitorenesduarte.github.io/page/other/ msc-thesis.pdf

Gilbert S, Lynch N (2002) Brewer's conjecture and the feasibility of consistent, available, partition-tolerant web services. SIGACT News 33(2):51-59, DOI http://doi.acm.org/10.1145/564585.564601

Gomes VBF, Kleppmann M, Mulligan DP, Beresford AR (2017) Verifying strong eventual consistency in distributed systems. Proc ACM Program Lang 1(OOPSLA):109:1-109:28, DOI 10.1145/3133933, URL http://doi.acm.org/ $10.1145 / 3133933$

Gonçalves RJT, Almeida PS, Baquero C, Fonte V (2017) DottedDB: Anti-Entropy without Merkle Trees, Deletes without Tombstones. In: Proceedings of the 2017 IEEE 36th Symposium on Reliable Distributed Systems (SRDS), pp 194-203, DOI 10.1109/SRDS.2017.28

Gotsman A, Yang H, Ferreira C, Najafzadeh M, Shapiro M (2016) 'cause i'm strong enough: Reasoning about consistency choices in distributed systems. In: Proceedings of the 43rd Annual ACM SIGPLAN-SIGACT Symposium on Principles of Programming Languages, ACM, New York, NY, USA, POPL '16, pp 371-384, DOI 10.1145/2837614.2837625, URL http://doi.acm.org/10.1145/ 2837614.2837625 
Kleppmann M, Beresford AR (2017) A conflict-free replicated json datatype. IEEE Transactions on Parallel and Distributed Systems 28(10):2733-2746, DOI 10. 1109/TPDS.2017.2697382

Kulkarni SS, Demirbas M, Madappa D, Avva B, Leone M (2014) Logical physical clocks. In: Aguilera MK, Querzoni L, Shapiro M (eds) Principles of Distributed Systems - 18th International Conference, OPODIS 2014, Cortina d'Ampezzo, Italy, December 16-19, 2014. Proceedings, Springer, Lecture Notes in Computer Science, vol 8878, pp 17-32, DOI 10.1007/978-3-319-14472-6_2, URL https: //doi.org/10.1007/978-3-319-14472-6_2

Lamport L (1978) Time, clocks, and the ordering of events in a distributed system. Commun ACM 21(7):558-565, DOI 10.1145/359545.359563, URL http://doi. acm.org/10.1145/359545.359563

Lamport L (1994) The temporal logic of actions. ACM Trans Program Lang Syst 16(3):872-923, DOI 10.1145/177492.177726, URL http://doi.acm.org/ $10.1145 / 177492.177726$

Li C, Porto D, Clement A, Gehrke J, Preguiça N, Rodrigues R (2012) Making georeplicated systems fast as possible, consistent when necessary. In: Proceedings of the 10th USENIX Conference on Operating Systems Design and Implementation, USENIX Association, Berkeley, CA, USA, OSDI'12, pp 265-278, URL http://dl.acm.org/citation.cfm?id=2387880.2387906

van der Linde A, Leitão Ja, Preguiça N (2016) $\Delta$-crdts: Making $\delta$-crdts deltabased. In: Proceedings of the $2 \mathrm{Nd}$ Workshop on the Principles and Practice of Consistency for Distributed Data, ACM, New York, NY, USA, PaPoC '16, pp 12:1-12:4, DOI 10.1145/2911151.2911163, URL http://doi.acm.org/10.1145/ 2911151.2911163

Malkhi D, Terry DB (2007) Concise version vectors in winfs. Distributed Computing 20(3):209-219, DOI 10.1007/s00446-007-0044-y, URL https://doi.org/10. 1007/s00446-007-0044-y 
O'Neil PE (1986) The escrow transactional method. ACM Trans Database Syst 11(4):405-430, DOI 10.1145/7239.7265, URL http://doi.acm.org/10.1145/ 7239.7265

Preguiça N, Marques JM, Shapiro M, Letia M (2009) A Commutative Replicated Data Type for Cooperative Editing. In: Proceedings of the 2009 29th IEEE International Conference on Distributed Computing Systems, IEEE Computer Society, Washington, DC, USA, ICDCS '09, pp 395-403, DOI 10.1109/ICDCS. 2009.20, URL http://dx.doi.org/10.1109/ICDCS.2009.20

Preguiça NM, Zawirski M, Bieniusa A, Duarte S, Balegas V, Baquero C, Shapiro M (2014) Swiftcloud: Fault-tolerant geo-replication integrated all the way to the client machine. In: 33rd IEEE International Symposium on Reliable Distributed Systems Workshops, SRDS Workshops 2014, Nara, Japan, October 6-9, 2014, IEEE Computer Society, pp 30-33, DOI 10.1109/SRDSW.2014.33, URL https: //doi.org/10.1109/SRDSW.2014.33

Roh HG, Jeon M, Kim JS, Lee J (2011) Replicated abstract data types: Building blocks for collaborative applications. J Parallel Distrib Comput 71(3):354-368, DOI 10.1016/j.jpdc.2010.12.006, URL http://dx.doi.org/10.1016/j.jpdc.2010. 12.006

Shapiro M, Preguiça N, Baquero C, Zawirski M (2011) Conflict-free replicated data types. In: Proceedings of the 13th International Conference on Stabilization, Safety, and Security of Distributed Systems, Springer-Verlag, Berlin, Heidelberg, SSS'11, pp 386-400, URL http://dl.acm.org/citation.cfm?id=2050613. 2050642

Sovran Y, Power R, Aguilera MK, Li J (2011) Transactional storage for georeplicated systems. In: Proceedings of the Twenty-Third ACM Symposium on Operating Systems Principles, ACM, New York, NY, USA, SOSP '11, pp 385-400, DOI 10.1145/2043556.2043592, URL http://doi.acm.org/10.1145/ 2043556.2043592 
Terry DB, Demers AJ, Petersen K, Spreitzer M, Theimer M, Welch BB (1994) Session guarantees for weakly consistent replicated data. In: Proceedings of the Third International Conference on Parallel and Distributed Information Systems (PDIS) 94, Austin, Texas, September 28-30, 1994, IEEE Computer Society, pp 140-149, DOI 10.1109/PDIS.1994.331722, URL https://doi.org/10.1109/ PDIS.1994.331722

Weiss S, Urso P, Molli P (2009) Logoot: A scalable optimistic replication algorithm for collaborative editing on p2p networks. In: Proceedings of the 2009 29th IEEE International Conference on Distributed Computing Systems, IEEE Computer Society, Washington, DC, USA, ICDCS '09, pp 404-412, DOI 10.1109/ICDCS.2009.75, URL http://dx.doi.org/10.1109/ICDCS.2009.75

Weiss S, Urso P, Molli P (2010) Logoot-undo: Distributed collaborative editing system on p2p networks. IEEE Trans Parallel Distrib Syst 21(8):1162-1174, DOI 10.1109/TPDS.2009.173, URL http://dx.doi.org/10.1109/TPDS.2009. 173

Zawirski M, Baquero C, Bieniusa A, Preguiça N, Shapiro M (2016) Eventually consistent register revisited. In: Proceedings of the $2 \mathrm{Nd}$ Workshop on the Principles and Practice of Consistency for Distributed Data, ACM, New York, NY, USA, PaPoC '16, pp 9:1-9:3, DOI 10.1145/2911151.2911157, URL http://doi.acm.org/10.1145/2911151.2911157

Zeller P (2017) Testing properties of weakly consistent programs with repliss. In: Proceedings of the 3rd International Workshop on Principles and Practice of Consistency for Distributed Data, ACM, New York, NY, USA, PaPoC'17, pp 3:1-3:5, DOI 10.1145/3064889.3064893, URL https://dl.acm.org/authorize? N37605

Zeller P, Bieniusa A, Poetzsch-Heffter A (2014) Formal specification and verification of crdts. In: Formal Techniques for Distributed Objects, FORTE 2014, Springer, Lecture Notes in Computer Science, pp 33-48 